TRANSACTIONS OF THE

AMERICAN MATHEMATICAL SOCIETY

Volume 169, July 1972

\title{
DIFFUSION AND BROWNIAN MOTION ON INFINITE-DIMENSIONAL MANIFOLDS $\left({ }^{1}\right)$
}

BY

\section{HUI-HSIUNG KUO}

\begin{abstract}
The purpose of this paper is to construct certain diffusion processes, in particular a Brownian motion, on a suitable kind of infinite-dimensional manifold. This manifold is a Banach manifold modelled on an abstract Wiener space. Roughly speaking, each tangent space $T_{x}$ is equipped with a norm and a densely defined inner product $g(x)$. Local diffusions are constructed first by solving stochastic differential equations. Then these local diffusions are pieced together in a certain way to get a global diffusion. The Brownian motion is completely determined by $g$ and its transition probabilities are proved to be invariant under

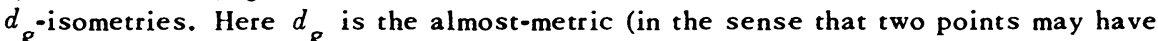
infinite distance) associated with $g$. The generalized Beltrami-Laplace operator is defined by means of the Brownian motion and will shed light on the study of potential theory over such a manifold.
\end{abstract}

I. Introduction. This paper is concerned with developing a natural integration theory over a certain type of Banach manifold. It is natural in the sense that this theory is associated with a Brownian motion. In [8] we took a step toward this goal by constructing local measures in a Banach manifold called RiemannWiener manifold. In this paper we use a different approach by considering stochastic differential equations on such a manifold. This idea stems from [6], [7] and [9]. We will construct a general class of diffusions which includes the Brownian motion as a special case.

Let $(i, H, B)$ be an abstract Wiener space [4] with $H$-norm denoted by $|\cdot|=$ $\langle\cdot, \cdot\rangle^{1 / 2}$ and $B$-norm by $\|\cdot\|$. It is important to keep in mind that $B^{*}$ is imbedded in $B$ so that $B^{*} \subset H \subset B$. (, ) will denote the natural pairing between $B^{*}$ and $B$. Note that $(x, y)=\langle x, y\rangle$ whenever $x$ is in $B^{*}$ and $y$ in $H . p_{t}$ denotes Wiener measure on $B$ with variance parameter $t>0$. We define for $x$ in $B$ and for a Borel subset $E$ of $B, p_{t}(x, E)=p_{t}(E-x)$. Fernique [3] has proved recently that $\int_{B} \exp \left\{\delta\|x\|^{2}\right\} p_{1}(d x)<\infty$ for some $\delta>0$.

We will assume the following on $(i, H, B):(1)\|\cdot\|$ is of class $C^{2}$ off the origin and (2) there exists an increasing sequence $Q_{n}$ of finite-dimensional projec-

Presented to the Society, April 10, 1970 under the title Beltrami-Laplace operator on Riemann-Wiener manifold; received by the editors May 24, 1971 and, in revised form, October 5, 1971.

AMS 1970 subject classifications. Primary 60J60, 60J65; Secondary 57A20, 58B20.

Key words and phrases. Abstract Wiener space, admissible transformation, BeltramiLaplace operator, Christoffel function, $d_{\mathcal{g}}$-isometry, Ito's formula, Riemann-Wiener manifold, spatially homogeneous, spur operator.

(1) Research supported by NSF Grant GP-19617. 
tions such that $Q_{n}(B) \subset B^{*}$ and $Q_{n}$ converges strongly to the identity both in $B$ and in $H$. Thus we can make use of the results in [8] and [9].

In this paper we will adopt the same notation used in [8]. We refer the reader to [8] for the following definitions. Let $(\hat{C}, \tau, g)$ be a $C^{k}$-Riemann-Wiener manifold $(k \geq 3)$ modelled on $(i, H, B)$. We will assume that $(\mathcal{C}$ is connected and separable. We assume also that, for any $\phi_{\alpha}, \phi_{\beta}$ in the admissible atlas, $\left\{\left(U_{\alpha}, \phi_{\alpha}\right) ; \alpha \epsilon\right.$ $\Lambda\}, \phi_{\beta}{ }^{\circ} \phi_{a}^{-1}$, in addition to being admissible, is assumed to be of at least class $C^{2}$ and to satisfy the following condition: $\left(\phi_{\beta} \circ \phi_{a}^{-1}\right)^{\prime \prime}(x)$, the second Fréchet derivative of $\phi_{\beta} \circ \phi_{a}^{-1}$ at $x$, belongs to $B\left(B, B ; B^{*}\right)$, the Banach space of all bounded bilinear maps from $B \times B$ into $B^{*}$ with norm

$$
\|\Phi\|_{B, B ; B^{*}}=\sup \left\{\|\Phi(u, v)\|_{B^{*}} /\|u\|\|v\| ; u \neq 0, v \neq 0, u, v \in B\right\},
$$

and $x \rightarrow\left(\phi_{\beta} \circ \phi_{\alpha}^{-1}\right)^{\prime \prime}(x)$ is continuous from $\phi_{\alpha}\left(U_{\alpha} \cap U_{\beta}\right)$ into $\mathfrak{B}\left(B, B ; B^{*}\right)$. The Christoffel function $\widetilde{\Gamma}$ is defined by

$$
\tilde{\Gamma}(x)(u, v)=1 / 2 \bar{g}(x)^{-1}\left\{g^{\prime}(x)(u, v, \cdot)+g^{\prime}(x)(v, \cdot, u)-g^{\prime}(x)(\cdot, u, v)\right\} .
$$

Thus $\widetilde{\Gamma}(x) \in \mathcal{B}\left(B, B ; B^{*}\right)$ for each $x$. Finally the local measures $\left\{q_{t}(x, \cdot) ; t>0\right.$, $x \in\{i\}$ are defined by $q_{t}(x, E)=p_{t}^{(x)}\left(0, \exp _{x}^{-1}(E)\right), E \in$ Borel field of $U(x)$, where $p_{t}^{(x)}$ is the Wiener measure in the tangent space $T_{x}\left(W^{0}\right)$ and $\exp _{x}$, the exponential map at $x$, is $C^{1}$-diffeomorphic in $U(x)$.

In SII we will make an estimation for admissible transformation. Also we will prove Ito's formula of the second type, regarding Ito's formula in [9] as the first type. \$III is devoted to the construction of certain diffusions in the RiemannWiener manifold by using the Ito-McKean technique ([6], [10]). In \$IV we study Brownian motion and its relation with the work of [8].

This paper is closely related to [1], although there are some technical differences between them. Furthermore, it is the author's conjecture that a Banach-Lie group is a Riemann-Wiener manifold. On the other hand, Eells and Elworthy [2] have recently developed Wiener integration on certain Banach manifolds by using a result in [8]. Roughly speaking, let $X_{W}$ be a Banach manifold modelled in $B$ with $C^{r}$-admissible atlas $\left\{\left(U_{i}, \phi_{i}\right)\right\}$. Let $g_{i j}$ be defined in $U_{i} \cap U_{j}$ by

$$
\begin{aligned}
g_{i j}(x)= & \exp \left\{(1 / 2 t)\left[-2\left\langle\phi_{j}(x)-\phi_{i}(x), \phi_{i}(x)\right\rangle-\left|\phi_{j}(x)-\phi_{i}(x)\right|^{2}\right\}\right. \\
& \times \operatorname{det}\left(\left(\phi_{j} \phi_{i}^{-1}\right)^{\prime}\left(\phi_{i}(x)\right)\right) .
\end{aligned}
$$

Then the family $\left\{g_{i j}\right\}_{i, j}$ forms the transition functions for a line bundle $W_{t}\left(X_{W}\right)$, which is called the bundle of Wiener densities over $X_{W}$ (with variance parameter $t$ ); the sections of $W_{t}\left(X_{W}\right)$ are called Wiener densities on $X_{W}$. Let $\xi$ be a Wiener density on $X_{W}$. Then they define a Borel measure $\mu(\xi)$ on $X_{W}$ by setting $\mu(\xi)(V)$ $=\int_{\phi_{i}(V)} \xi_{i}(x) d p_{t}(x)$ for any open set $V$ in $U_{i}$, where $p_{t}$ is the Wiener measure of 
$B$ with variance parameter $t$. They have succeeded in connecting this kind of integration theory with degree theory. However, their point of view is different from ours.

We would like to thank Professor H. P. McKean for his valuable conversations and suggestions.

II. Admissible transformation and Ito's formula of the second type. Let $U$ and $V$ be open subsets of $B$. In [8] we define a homeomorphism $T$ from $U$ onto $V$ to be admissible if $T$ is a $C^{1}$-diffeomorphism and $T x-x \in H, T^{\prime}(x)-I \epsilon$ $\mathcal{B}\left(B, B^{*}\right)$ for all $x \in U$, and the map $x \rightarrow T^{\prime}(x)-I$ is continuous from $U$ into $\mathcal{B}\left(B, B^{*}\right)$. Here $\mathcal{B}\left(B, B^{*}\right)$ denotes the Banach space of all bounded operators from $B$ into $B^{*}$ with norm $\|S\|_{B, B^{*}}=\sup \left\{\|S u\|_{B^{*}} /\|u\| ; u \neq 0, u \in B\right\}$.

Proposition II.1. Let $T$ be admissible on an open set $U$ containing the origin and let $T(0)=0, T^{\prime}(0)=I$. Assume $T^{\prime \prime}(x) \in \mathfrak{B}\left(B, B ; B^{*}\right)$ for all $x \in U$ and let $T^{\prime \prime}$ be continuous from $U$ into $B\left(B, B ; B^{*}\right)$. Then there exists $r>0$ such that $b(0, r,\|\cdot\|) \equiv\{x \in B ;\|x\|<r\} \subset U$ and

$$
\left|p_{t}(T(E))-p_{t}(E)\right| \leq M \sqrt{t}
$$

bolds for all $t>0$, all Borel subsets $E$ of $b(0, r,\|\cdot\|)$. M is a constant independent of $t$ and $E$.

Proof. Let $K=T-I$. Choose $r>0$ small enough to meet the conditions

(i) $b(0, r,\|\cdot\|) \subset U \cap b(0,1,\|\cdot\|)$,

(ii) $\left\|K^{\prime \prime}(x)\right\|_{B, B ; B^{*}} \leq 1+\left\|K^{\prime \prime}(0)\right\|_{B, B ; B^{*}}$ for all $x \in b(0, r,\|\cdot\|)$, and

(iii) $2 r\left(1+\left\|K^{\prime \prime}(0)\right\|_{B, B ; B^{*}}\right)<\delta$, where $\delta>0$ is such that $\int_{B} \exp \left\{\delta\|x\|^{2}\right\}_{p_{1}}(d x)<\infty$,

(iv) $|\operatorname{det}| T^{\prime}(x)|-1| \leq c\|x\|$ for all $x \in b(0, r,\|\cdot\|)$, where $c$ is a finite constant independent of $x$.

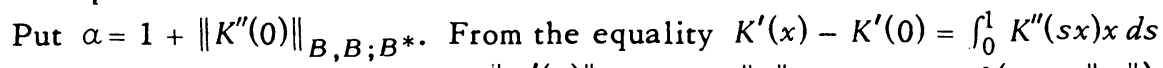
and (ii) we obtain immediately that $\left\|K^{\prime}(x)\right\|_{B, B^{*}} \leq \alpha\|x\|$ for all $x \in b(0, r,\|\cdot\|)$. Similarly, $\|K(x)\|_{B^{*}} \leq a\|x\|^{2}$ for all $x \in b(0, r,\|\cdot\|)$. Therefore for all $0 \leq s \leq 1$ and all $x \in b(0, r,\|\cdot\|)$ we have

$$
\begin{aligned}
|(K(s x), x)| & \leq \alpha\|x\|^{3}, \\
\left|\left(K^{\prime}(s x) x, s x\right)\right| & \leq \alpha\|x\|^{3}, \\
\left|\left(K(s x), K^{\prime}(s x) x\right)\right| & \leq \beta^{2} \alpha^{2}\|x\|^{4} \leq \beta^{2} \alpha^{2}\|x\|^{3},
\end{aligned}
$$

where $\beta$ is some constant such that $\|x\| \leq \beta|x|$ for all $x$ in $H$.

Now define a Borel measure $\psi_{t}(d x)$ on $b(0, r,\|\cdot\|)$ by $\psi_{t}(d x)=b_{t}(x) p_{t}(d x)$, where $b_{t}(x)=\exp \left\{\left[-2(K x, x)-|K x|^{2}\right] / 2 t\right\}$. Let $E$ be any fixed Borel subset of $b(0, r,\|\cdot\|)$. Then 


$$
\begin{aligned}
\psi_{t}(E) & =\int_{E} b_{t}(x) p_{t}(d x) \\
& =\int_{E}\left\{b_{t}(0)+\int_{0}^{1}\left(b_{t}^{\prime}(s x), x\right) d s\right\} p_{t}(d x) \\
& =p_{t}(E)+\int_{0}^{1} d s \int_{E}\left(b_{t}^{\prime}(s x), x\right) p_{t}(d x) .
\end{aligned}
$$

Thus

$$
\psi_{t}(E)-p_{t}(E)=\int_{0}^{1} d s \int_{E}\left(b_{t}^{\prime}(s x), x\right) p_{t}(d x) .
$$

It is easy to check that

$$
\left(h_{t}^{\prime}(s x), x\right)=-t^{-1}\left[(K(s x), x)+\left(K^{\prime}(s x), s x\right)+\left(K(s x), K^{\prime}(s x) x\right)\right] b_{t}(s x) \text {. }
$$

Thus from (1) we have

$$
\left|\left(b_{t}^{\prime}(s x), x\right)\right| \leq t^{-1}\left(2 \alpha+\beta^{2} \alpha^{2}\right)\|x\|^{3} \exp \left\{t^{-1} \alpha\|x\|^{3}\right\} .
$$

Putting (3) into (2), we get immediately

$$
\left|\psi_{t}(E)-p_{t}(E)\right| \leq t^{-1}\left(2 \alpha+\beta^{2} \alpha^{2}\right) \int_{E}\|x\|^{3} \exp \left\{t^{-1} \alpha\|x\|^{3}\right\}_{p_{t}}(d x)
$$

$$
\leq t^{-1}\left(2 \alpha+\beta^{2} \alpha^{2}\right)\left[\int_{E}\|x\|^{6} p_{t}(d x)\right]^{1 / 2}\left[\int_{E} \exp \left\{2 t^{-1} \alpha\|x\|^{3}\right\} p_{t}(d x)\right]^{1 / 2} .
$$

But $\int_{E}\|x\|^{6} p_{t}(d x) \leq t^{3} \int_{B}\|x\|^{6} p_{1}(d x)$ and

$$
\begin{aligned}
\int_{E} \exp \left\{2 t^{-1}\right. & \left.\alpha\|x\|^{3}\right\} p_{t}(d x) \leq \int_{E} \exp \left\{2 t^{-1} r \alpha\|x\|^{2}\right\} p_{t}(d x) \\
& \leq \int_{E} \exp \left\{\delta t^{-1}\|x\|^{2}\right\} p_{t}(d x) \leq \int_{B} \exp \left\{\delta t^{-1}\|x\|^{2}\right\}_{p_{t}}(d x) \\
& =\int_{B} \exp \left\{\delta\|x\|^{2}\right\} p_{1}(d x) .
\end{aligned}
$$

Here we have made the change of variable $x / \sqrt{t} \rightarrow x$ in passing from $p_{t}(d x)$ to $p_{1}(d x)$. We have also used (iii). Putting these estimates into (4), we get

(5) $\left|\psi_{t}(E)-p_{t}(E)\right| \leq \sqrt{t}\left(2 \alpha+\beta^{2} \alpha^{2}\right)\left[\int_{B}\|x\|^{6} p_{1}(d x) \int_{B} \exp \left\{\delta\|x\|^{2}\right\}_{p_{1}}(d x)\right]^{1 / 2}$.

On the other hand, from Theorem I.4 of [8], we know $p_{t}(T(E))$ $=\int_{E} b_{t}(x) \operatorname{det}\left|T^{\prime}(x)\right| p_{t}(d x)$. Therefore

$$
\begin{aligned}
\left|p_{t}(T(E))-\psi_{t}(E)\right| & \leq \int_{E} b_{t}(x)|\operatorname{det}| T^{\prime}(x)|-1| p_{t}(d x) \\
& \leq c \int_{E} b_{t}(x)\|x\| p_{t}(d x) \text { by (iv). }
\end{aligned}
$$

The same argument as before yields

$$
\left|p_{t}(T(E))-\psi_{t}(E)\right| \leq c \sqrt{t}\left[\int_{B}\|x\|^{2} p_{1}(d x) \int_{B} \exp \left\{\delta\|x\|^{2}\right\} p_{1}(d x)\right]^{1 / 2} .
$$

Obviously, (5) and (6) give the desired conclusion. Q.E.D. 
The Ito formula we prove in [9] answers the following question: Given $d X(t)$ $=\xi(t) d W(t)+\sigma(t) d t$, where $W(t)$ is a Wiener process in $B, \xi$ is an n.a.t. and $\sigma$ is an n.a.v. (see [9] for the definitions), and a real-valued function $f$ on $B$ with certain regularity, then what is $d f(X(t))$ ? Now we ask another question: Given $d X(t)=\xi(t) d W(t)+\sigma(t) d t$ and a map $\theta$ from $B$ into itself with certain regularity, then what is $d \theta(X(t))$ ? We will prove a formula in Theorem II.1 to answer this question. It will turn out that $d \theta(X(t))$ has an expression similar to $d X(t)$. In order to state the formula we have to make the following

Definition II.1. A continuous bilinear map $\Phi$ from $H \times H$ into $H$ is called a spur operator if (i) for all $b \in H, \Phi_{b} \in \mathcal{B}_{1}(H, H)$, the Banach space of all trace class operators, where $\Phi_{b}(u, v)=\langle\Phi(u, v), b\rangle$ and (ii) the linear functional $b \rightarrow$ trace $\Phi_{b}$ is continuous.

Notation. It follows from the definition that there exists a unique element $b_{0}$ in $H$ such that $\left\langle b_{0}, b\right\rangle=\operatorname{trace} \Phi_{b}$ for all $b$ in $H$. We denote this unique element $b_{0}$ by sp $\Phi$. The vector space of all spur operators in $H$ will be denoted by $\mathcal{S}(H)$.

Proposition II.2. (i) If $\Phi \in \mathcal{S}(H)$ and $\left\{e_{k}\right\}_{k=1}^{\infty}$ is an orthonormal basis of $H$ then the series $\sum_{k=1}^{\infty} \Phi\left(e_{k}, e_{k}\right)$ converges in $H$. Moreover, $\sum_{k=1}^{\infty} \Phi\left(e_{k}, e_{k}\right)=\operatorname{sp} \Phi$.

(ii) $\mathfrak{B}\left(B, B ; B^{*}\right) \subset S^{(}(H)$, i.e. a continuous bilinear map from $B \times B$ into $B^{*}$ is a spur operator when it is restricted to $H$.

(iii) If $\Phi \in \mathcal{S}(H)$ and $S, T$ are continuous linear operators of $H$ then $\Phi \circ$ $(S \times T)$ and $S \circ \Phi$ belong to $\mathcal{S}(H)$ and $\operatorname{sp} S \circ \Phi=S(\operatorname{sp} \Phi)$.

Proof. (i) and (iii) are easy, while (ii) follows from Proposition 0.1 of [8]. Q.E.D.

Theorem II.1 (Ito's formula of the second type). Let $\theta$ be a $C^{2}$-map from $B$ into itself such that (i) $\theta^{\prime}(x)-I \in \mathcal{B}\left(B, B^{*}\right), \theta^{\prime \prime}(x) \in \mathcal{B}\left(B, B ; B^{*}\right)$ for all $x$ in $B$, and (ii) the maps $x \rightarrow \theta^{\prime}(x)-I, x \rightarrow \theta^{\prime \prime}(x)$ are continuous from $B$ into $\mathcal{B}\left(B, B^{*}\right)$, $\mathfrak{B}\left(B, B ; B^{*}\right)$, respectively.

If $X(t)=x_{0}+\int_{0}^{t} \xi(s) d W(s)+\int_{0}^{t} \sigma(s) d s$, where $\xi$ is a nonanticipating transformation and $\sigma$ is a nonanticipating vector (see [9] for the definitions), then

$$
\begin{aligned}
\theta(X(t))= & \theta\left(x_{0}\right)+\int_{0}^{t} \theta^{\prime}(X(s)) \circ \xi(s) d W(s) \\
& +\int_{0}^{t}\left\{\theta^{\prime}(X(s))(\sigma(s))+1 / 2 \operatorname{sp} \theta^{\prime \prime}(X(s)) \circ[\xi(s) \times \xi(s)]\right\} d s .
\end{aligned}
$$

Proof. Let $b$ be any element in $B^{*}$ and define $f(x)=(\theta(x), b)$. Then $f^{\prime}(x)=$ $\theta^{\prime}(x)^{*} b$ and $f^{\prime \prime}(x) \in \mathfrak{B}\left(B, B^{*}\right)$ is given by $\left(f^{\prime \prime}(x) u, v\right)=\left(\theta^{\prime \prime}(x)(u, v), b\right), u, v \in B$. Here the star indicates the adjoint operator with respect to $H$, i.e. $\theta^{\prime}(x)^{*}$ means $\left(\left.\theta^{\prime}(x)\right|_{H}\right)^{*}$, where $\left.\theta^{\prime}(x)\right|_{H}: H \rightarrow H$. Note that by the assumption on $\theta$ it follows that $f^{\prime}(x) \in B^{*}$ and $f^{\prime \prime}(x) \in \mathfrak{B}\left(B, B^{*}\right)$ for all $x$. Thus we can apply Theorem 4.2 of [9] to get 


$$
\begin{aligned}
f(X(t))= & f\left(x_{0}\right)+\int_{0}^{t}\left(\xi^{*}(s) f^{\prime}(X(s)), d W(s)\right) \\
& +\int_{0}^{t}\left\{\left\langle f^{\prime}(X(s)), \sigma(s)\right\rangle+1 / 2 \text { trace } \xi^{*}(s) f^{\prime \prime}(X(s)) \xi(s)\right\} d s .
\end{aligned}
$$

But

$$
\int_{0}^{t}\left(\xi^{*}(s) f^{\prime}(X(s)), d W(s)\right)=\int_{0}^{t}\left(\xi^{*}(s) \circ \theta^{\prime}(X(s)) * b, d W(s)\right)
$$

$$
\begin{gathered}
=\left(\int_{0}^{t} \theta^{\prime}(X(s)) \circ \xi(s) d W(s), b\right), \\
\int_{0}^{t}\left\langle f^{\prime}(X(s)), \sigma(s)\right\rangle d s=\int_{0}^{t}\left\langle\theta^{\prime}(X(s)) * b, \sigma(s)\right\rangle d s \\
=\int_{0}^{t}\left\langle\theta^{\prime}(X(s))(\sigma(s)), b\right\rangle d s=\left\langle\int_{0}^{t} \theta^{\prime}(X(s))(\sigma(s)) d s, b\right\rangle .
\end{gathered}
$$

Now, consider the operator $\xi^{*}(s) f^{\prime \prime}(X(s)) \xi(s)$ from $H$ into itself. Let $u$, $v$ be in H. Then

$$
\begin{aligned}
\left\langle\xi^{*}(s) f^{\prime \prime}(X(s)) \xi(s) u, v\right\rangle=\left\langle f^{\prime \prime}(X(s)) \xi(s) u, \xi(s) v\right\rangle & \\
& =\left(f^{\prime \prime}(X(s)) \xi(s) u, \xi(s) v\right)=\left(\theta^{\prime \prime}(X(s))(\xi(s) u, \xi(s) v), b\right) \\
& =\left\langle\theta^{\prime \prime}(X(s)) \circ[\xi(s) \times \xi(s)](u, v), k\right\rangle=\Phi_{b}(u, v),
\end{aligned}
$$

where $\Phi=\theta^{\prime \prime}(X(s)) \circ[\xi(s) \times \xi(s)]$.

Note that by the assumption on $\theta$ and (ii), (iii) of Proposition II. 2 it follows that $\Phi \in \mathfrak{S}(H)$. Therefore,

$$
\begin{gathered}
\operatorname{trace} \xi^{*}(s) f^{\prime \prime}(X(s)) \xi(s)=\operatorname{trace} \Phi_{b}=\langle s p \Phi, b\rangle \\
=\left\langle\operatorname{sp} \theta^{\prime \prime}(X(s)) \circ[\xi(s) \times \xi(s)], b\right\rangle .
\end{gathered}
$$

Putting (8), (9) and (10) into (7), we get

$$
\begin{aligned}
(\theta(X(t)), b)= & \left(\theta\left(x_{0}\right), b\right)+\left(\int_{0}^{t} \theta^{\prime}(X(s)) \circ \xi(s) d W(s), b\right) \\
& +\left\langle\int_{0}^{t}\left\{\theta^{\prime}(X(s))(\sigma(s))+1 / 2 \operatorname{sp} \theta^{\prime \prime}(X(s)) \circ[\xi(s) \times \xi(s)]\right\} d s, b\right\rangle,
\end{aligned}
$$

for all $b \in B^{*}$. Note that $\langle x, y\rangle=(x, y)$, whenever $x \in B^{*}$ and $y \in H$. Then the formula of the theorem folows easily. Q.E.D.

III. Construction of diffusions on a Riemann-Wiener manifold. Let $((\mathbb{8}, \tau, g)$ be a connected, separable $C^{k}$-Riemann-Wiener manifold $(k \geq 3)$. Suppose for each chart $(U, \phi)$ in $C i$ we are given two maps $A_{\phi}$ and $\sigma_{\phi}$ from $\phi(U) \subset B$ into $\mathfrak{B}(B, B)$ and $H$, respectively, such that $A_{\phi}(x)-I \in \mathcal{B}\left(B, B^{*}\right)$ and $A_{\phi}(x)$ is nonsingular for all $x \in \phi(U)$.

Let $K$ be a continuous linear operator from $B$ into $B^{*}$. Then the restriction $\left.K\right|_{H}$ of $K$ to $H$ is a continuous linear operator from $H$ into itself. Let $\left(\left.K\right|_{H}\right)^{*}$ be the adjoint operator of $\left.K\right|_{H}$. It is easy to check that $\left\|\left(\left.K\right|_{H}\right)^{*} b\right\|_{B} \leq\|K\|_{B, B^{*}}\|b\|$ 
for all $b$ in $H$. Therefore there exists a unique continuous extension $\left(K_{H}\right)^{*}$ of $\left(\left.K\right|_{H}\right)^{*}$ to $B$. Obviously $\left(\left.K\right|_{H}\right) *(B) \subset B^{*}$. In fact it can be checked that $\|\left(\widetilde{\left.\left.K\right|_{H}\right)^{*}}\left\|_{B, B^{*}}=\right\| K \|_{B, B^{*}}\right.$. Suppose $T$ is a continuous linear operator of $B$ such that $(T-I)(B) \subset B^{*}$. Then we define $T^{*}=I+\left((T-I) T_{H}\right)^{*}$. Clearly $T^{*}-I \epsilon$ $B\left(B, B^{*}\right)$.

Definition III.1. By diffusion coefficients in 60 we mean a pair $\left(A_{\phi}, \sigma_{\phi}\right)$ for each chart $(U, \phi)$ of the above maps satisfying the transformation rules: If $(U, \phi)$ and $(V, \psi)$ are two charts with $U \cap V \neq \emptyset$ then

$$
\left\{\begin{array}{l}
A_{\psi}(\bar{x}) A_{\psi}(\bar{x}) *=\theta^{\prime}(x) A_{\phi}(x) A_{\phi}(x)^{*} \theta^{\prime}(x)^{*}, \\
\sigma_{\psi}(\bar{x})=\theta^{\prime}(x)\left(\sigma_{\phi}(x)\right)+1 / 2 \operatorname{sp} \theta^{\prime \prime}(x) \circ\left[A_{\phi}(x) \times A_{\phi}(x)\right],
\end{array}\right.
$$

where $\theta=\psi \circ \phi^{-1}$ and $\bar{x}=\theta(x)$.

Remark 1. By assumption $\theta^{\prime}(x)-I \in \mathfrak{B}\left(B, B^{*}\right)$ and $\theta^{\prime \prime}(x) \in \mathfrak{B}\left(B, B ; B^{*}\right)$. Thus $\theta^{\prime}(x) A_{\phi}(x) A_{\phi}(x)^{*} \theta^{\prime}(x)^{*}-I \in \mathcal{B}\left(B, B^{*}\right)$ which is consistent with $A_{\psi}(\bar{x}) A_{\psi}(\bar{x})^{*}-I \epsilon$ $\mathfrak{B}\left(B, B^{*}\right)$. Moreover, $\theta^{\prime \prime}(x) \circ\left[A_{\phi}(x) \times A_{\phi}(x)\right] \in \mathfrak{S}(H)$ by (ii) and (iii) of Proposition II.2. Thus sp $\theta^{\prime \prime}(x) \circ\left[A_{\phi}(x) \times A_{\phi}(x)\right] \in H$. Note also that $\theta^{\prime}(x)(H) \subset H$, so $\theta^{\prime}(x)\left(\sigma_{\phi}(x)\right) \in H$ for all $x \in \phi(U \cap V)$.

Remark 2. For each $x \in \phi(U \cap V)$ there exists a bounded linear operator $S_{\phi}(x)$ of $B$ such that $S_{\phi}(x)-I \in \mathcal{B}\left(B, B^{*}\right)$ and $\left.S_{\phi}(x)\right|_{H}$ is a unitary operator of $H$ and $A_{\psi}(\bar{x})=\theta^{\prime}(x) \circ A_{\phi}(x) \circ S_{\phi}(x)$. To see this simply put $S_{\phi}(x)=A_{\phi}(x)^{-1} \circ$ $\theta^{\prime}(x)^{-1} \circ A_{\psi}(\bar{x})$ and use the transformation rule.

Remark 3. From now on we will drop the indices in charts in case there is no confusion, for instance, when we are considering the chart $(U, \phi)$ and $x \in \phi(U)$ then $A(x)$ and $S(x)$ mean $A_{\phi}(x)$ and $S_{\phi}(X)$, respectively. Similarly, if $(V, \psi)$ is another chart such that $U \cap V \neq \emptyset$ and $\bar{x} \in \psi(U \cap V)$ then $A(\bar{x})$ means $A_{\psi}(\bar{x})$.

Recall that in the Riemann-Wiener manifold 6 we have the Riemannian structure $g$. For each $x$ in $6, g(x)$ is a positive definite symmetric bilinear form of $H$. Thus the corresponding operator $\bar{g}(x)$ of $g(x)$ (i.e. $\langle\bar{g}(x) h, k\rangle=g(x)(b, k)$ for $b, k \in H)$ is a selfadjoint positive definite operator of $H$. Hence the inverse $\bar{g}(x)^{-1}$ of $\bar{g}(x)$ is also selfadjoint and positive definite. Let $\bar{g}(x)^{-1 / 2}$ denote the selfadjoint positive definite square root of $\bar{g}(x)^{-1}$. It follows from the assumption on $g$ (namely, RW-3, p. 69 of [8]) that $\bar{g}(x)$ is of the form $I_{H}+K(x)$, where $K(x) \epsilon$ $\mathfrak{B}\left(H_{0}, B^{*}\right)$. Here we have used $I_{H}$ temporarily to indicate the identity map of $H$ for the sake of emphasis. $H_{0}$ denotes the normed linear space $(H,\|\cdot\|)$. It is easy to check that $\bar{g}(x)^{-1 / 2}-I_{H} \in \mathcal{B}\left(H_{0}, B^{*}\right)$. Let $\left(\bar{g}(x)^{-1 / 2}-I_{H}\right)^{\sim}$ be the extension of $\bar{g}(x)^{-1 / 2}-I_{H}$ to $B$. Thus $\left(\bar{g}(x)^{-1 / 2}-I_{H}\right)^{\sim} \in \mathscr{B}\left(B, B^{*}\right)$. We will use the same notation $\vec{g}(x)^{-1 / 2}$ to denote $I_{B}+\left(\bar{g}(x)^{-1 / 2}-I_{H}\right)^{2}$ because there is no confusion. Therefore $\bar{g}(x)^{-1 / 2} \in \mathcal{B}(B, B)$ and $\bar{g}(x)^{-1 / 2}-l \in \mathfrak{B}\left(B, B^{*}\right)$.

On the other hand, Proposition II. 2 tells us that $\widetilde{\Gamma}(x) \circ\left[\bar{g}(x)^{-1 / 2} \times \bar{g}(x)^{-1 / 2}\right] \epsilon$ $\mathcal{S}(H)$ because $\widetilde{\Gamma}(x) \in \mathfrak{B}\left(B, B ; B^{*}\right)$ and $\bar{g}(x)^{-1 / 2} \in \mathcal{B}(B, B)$. Hence sp $\widetilde{\Gamma}(x) \circ$ $\left[\bar{g}(x)^{-1 / 2} \times \bar{g}(x)^{-1 / 2}\right] \in H$. Now we are ready to show the following 
Proposition III. 1. $A(x)=\bar{g}(x)^{-1 / 2}$ and $\sigma(x)=-1 / 2$ sp $\widetilde{\Gamma}(x) \circ\left[\bar{g}(x)^{-1 / 2} \times \bar{g}(x)^{-1 / 2}\right]$ are diffusion coefficients.

Proof. We need only to show that $A$ and $\sigma$ satisfy the transformation rule (11).

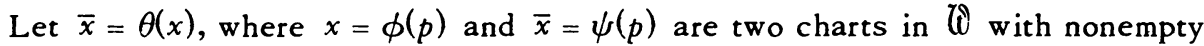
common domain. By definition $\langle\bar{g}(x) u, v\rangle=g(p)\left(\phi_{*, p}^{-1} u, \phi_{*, p}^{-1} v\right)$ and $\langle\bar{g}(\bar{x}) u, v\rangle=$ $g(p)\left(\psi_{*, p}^{-1} u, \psi_{*, p}^{-1} v\right)$ for all $u, v \in H$. It follows that $\left\langle\bar{g}(\bar{x}) \theta^{\prime}(x) u, \theta^{\prime}(x) v\right\rangle=$ $\langle\bar{g}(x) u, v\rangle$ for all $u, v \in H$. Therefore

$$
\bar{g}(\bar{x})=\left(\theta^{\prime}(x)^{*}\right)^{-1} \bar{g}(x) \theta^{\prime}(x)^{-1} \text {. }
$$

(12) implies that $\bar{g}(\bar{x})^{-1}=\theta^{\prime}(x) \bar{g}(x)^{-1} \theta^{\prime}(x)^{*}$, which is the first of the transformation rules (11).

On the other hand, it follows from (12) by a simple computation that, for all $u, v, w \in B$,

$$
\begin{aligned}
g^{\prime}(\bar{x})(u, v, w)= & g^{\prime}(x)\left(\theta^{\prime}(x)^{-1} u, \theta^{\prime}(x)^{-1} v, \theta^{\prime}(x)^{-1} w\right) \\
& -\left\langle\theta^{\prime \prime}(x)\left(\theta^{\prime}(x)^{-1} u, \theta^{\prime}(x)^{-1} v\right), \bar{g}(\bar{x}) w\right\rangle \\
& -\left\langle\theta^{\prime \prime}(x)\left(\theta^{\prime}(x)^{-1} u, \theta^{\prime}(x)^{-1} w\right), \ddot{g}(\bar{x}) v\right\rangle .
\end{aligned}
$$

Recall that the Christoffel function $\widetilde{\Gamma}$ is defined as follows: For all $u, v \in B$,

$$
\widetilde{\Gamma}(\bar{x})(u, v)=1 / 2 \bar{g}(\bar{x})^{-1}\left\{g^{\prime}(\bar{x})(u, v, \cdot)+g^{\prime}(\bar{x})(v, \cdot u)-g^{\prime}(\bar{x})(\cdot, u, v)\right\} .
$$

In particular, for all $u \in B$,

(14) $\widetilde{\Gamma}(\bar{x})(u, u)=1 / 2 \bar{g}(\bar{x})^{-1}\left\{g^{\prime}(\bar{x})(u, u, \cdot)+g^{\prime}(\bar{x})(u, \cdot, u)-g^{\prime}(\bar{x})(\cdot, u, u)\right\}$.

If (12) and (13) are put into (14), an easy computation shows that, for all $b \in H$,

$$
\begin{aligned}
\widetilde{\Gamma}(\bar{x}) \circ\left[\bar{g}(\bar{x})^{-1 / 2} \times \bar{g}(\bar{x})^{-1 / 2}\right](b, b) \\
=\theta^{\prime}(x) \circ \widetilde{\Gamma}(x) \circ\left[\bar{g}(x)^{-1 / 2} \times \bar{g}(x)^{-1 / 2}\right] \circ[S(x) \times S(x)](b, b) \\
\quad-\theta^{\prime \prime}(x) \circ\left[\bar{g}(x)^{-1 / 2} \times \bar{g}(x)^{-1 / 2}\right] \circ[S(x) \times S(x)](b, b),
\end{aligned}
$$

where $S(x)$ is given by $\bar{g}(\bar{x})^{-1 / 2}=\theta^{\prime}(x) \bar{g}(x)^{-1 / 2} S(x)$ as in Remark 2 following Definition III.1. Note that the three bilinear maps from $H \times H$ into $H$ in the above equality are all symmetric. Therefore,

$$
\begin{aligned}
\widetilde{\Gamma}(\bar{x}) \circ\left[\bar{g}(\bar{x})^{-1 / 2} \times \bar{g}(\bar{x})^{-1 / 2}\right]= & \theta^{\prime}(x) \circ \widetilde{\Gamma}(x) \circ\left[\bar{g}(x)^{-1 / 2} \times \bar{g}(x)^{-1 / 2}\right] \circ[S(x) \times S(x)] \\
& -\theta^{\prime \prime}(x) \circ\left[\bar{g}(x)^{-1 / 2} \times \bar{g}(x)^{-1 / 2}\right] \circ[S(x) \times S(x)] .
\end{aligned}
$$

Taking sp on both sides and noting that

$$
\begin{aligned}
1 / 2 \operatorname{sp} & \theta^{\prime}(x) \circ \widetilde{\Gamma}(x) \circ\left[\bar{g}(x)^{-1 / 2} \times \bar{g}(x)^{-1 / 2}\right] \circ[S(x) \times S(x)] \\
= & \theta^{\prime}(x)\left(1 / 2 \operatorname{sp} \widetilde{\Gamma}(x) \circ\left[\bar{g}(x)^{-1 / 2} \times \bar{g}(x)^{-1 / 2}\right] \circ[S(x) \times S(x)]\right) \\
= & \theta^{\prime}(x)\left(1 / 2 \operatorname{sp} \tilde{\Gamma}(x) \circ\left[\bar{g}(x)^{-1 / 2} \times \bar{g}(x)^{-1 / 2}\right]\right)
\end{aligned}
$$

since $S(x)$ is unitary (see (i) of Proposition II.2), we end up with 


$$
\begin{aligned}
\sigma(\bar{x}) & =\theta^{\prime}(x)(\sigma(x))+1 / 2 \operatorname{sp} \theta^{\prime \prime}(x) \circ\left[\bar{g}(x)^{-1 / 2} \times \bar{g}(x)^{-1 / 2}\right] \circ[S(x) \times S(x)] \\
& =\theta^{\prime}(x)(\sigma(x))+1 / 2 \operatorname{sp} \theta^{\prime \prime}(x) \circ\left[\bar{g}(x)^{-1 / 2} \times \bar{g}(x)^{-1 / 2}\right]
\end{aligned}
$$

which is the second of the transformation rules (11). Q.E.D.

Definition III.2. Diffusion coefficients $(A, \sigma)$ are locally Lipschitzian if for each point $p$ in there exist a chart $(U, \phi)$ at $p$ and a constant $\alpha(p)$ depending only on $p$ such that, for all $x$ and $y$ in $\phi(U)$,

$$
\begin{gathered}
\|A(x)-A(y)\|_{2} \leq \alpha(p)\|x-y\|, \\
|\sigma(x)-\sigma(y)| \leq \alpha(p)\|x-y\| .
\end{gathered}
$$

Remark. $\|\cdot\|_{2}$ denotes the Hilbert-Schmidt norm. Note that $A(x)-I \epsilon$ $\mathfrak{B}\left(B, B^{*}\right) \subset \mathfrak{B}_{1}(H, H) \subset \mathfrak{B}_{2}(H, H)$ for all $x \in \phi(U)$. Thus $A(x)-A(y) \in \mathfrak{B}_{2}(H, H)$ for all $x$ and $y$ in $\phi(U)$.

Let $(A, \sigma)$ be fixed locally Lipschitzian diffusion coefficients. We will solve the stochastic differential equation $d \mathfrak{X}(t, \omega)=A(\mathfrak{X}(t, \omega)) d W(t, \omega)+\sigma(\mathfrak{X}(t, \omega)) d t$ to get a diffusion $\mathfrak{X}(t)$ in the manifold $t^{0}$ with infinitesimal generator arising from $(A, \sigma)$.

(A) Local diffusions. We use $p$ to denote a generic point of 60 . Let $(V(p)$, $\left.\phi_{(p)}\right)$ denote a chart at $p$ such that $\phi_{(p)}(V(p)) \subset B$ is an open ball around $\phi_{(p)}(p)$ and the pair $(A, \sigma)$ is Lipschitzian in $\phi_{(p)}(V(p))$. This can always be done by choosing a smaller neighborhood at $p$, if necessary. Let $W(p)$ denote the open neighborhood of $p$ such that $\phi_{(p)}(W(p))$ is an open ball around $\phi_{(p)}(p)$ with radius half of that of $\phi_{(p)}(V(p))$. Recall that $U(p)$ denotes an open neighborhood at $p$ where $\exp _{p}$, the exponential map at $p$, is a $C^{k-2}$-diffeomorphism $(k \geq 3)$. This notation will be used throughout the rest of this paper.

Let $\lambda(x)$ be a $C^{1}$-function from $B$ into $[0,1]$ such that

Define

$$
\begin{aligned}
\lambda(x)=1 & \text { if } x \in \phi_{(p)}(W(p)), \\
\lambda(x)=0 & \text { if } x \notin \phi_{(p)}(V(p)), \\
\left\|\lambda^{\prime}(x)\right\|_{B^{*}} \leq 1 & \text { for all } x \in B .
\end{aligned}
$$

$$
\widetilde{A}(x)=I+\lambda(x)(A(x)-I), \quad \widetilde{\sigma}(x)=\lambda(x) \sigma(x) .
$$

Then $\tilde{A}$ and $\widetilde{\sigma}$ are globally defined in $B$ and $\widetilde{A}=A, \widetilde{\sigma}=\sigma$ on $\phi_{(p)}(W(p))$. Moreover, $\tilde{A}$ and $\tilde{\sigma}$ satisfy the hypothesis of Theorem 5.1 in [9]. Note that instead of defining $\widetilde{A}(x)=\lambda(x) A(x)$, we define $\widetilde{A}(x)$ as above in order to meet the assumption of Theorem 5.1 in [9]. Therefore by its conclusion the stochastic integral equation

$$
X(t)=x_{0}+\int_{0}^{t} \tilde{A}(X(s)) d W(s)+\int_{0}^{t} \tilde{\sigma}(X(s)) d s
$$

has a unique continuous solution, where $x_{0} \in \phi_{(p)}(W(p))$. 
Let $\rho$ be the exit time of $X(t)$ from $\phi_{(p)}(W(p))$. Then the local diffusion $X_{1}(t)=X(t \wedge \rho)$ begins afresh at Brownian stopping times (see [10] for the meaning) and does not depend on the mode of extension of $A$ and $\sigma$.

(B) Global diffusion. Obviously $\left\{W(p) ; p \in[Q\}\right.$ is a covering on 6 . Let $d_{\tau}$ denote the metric in 60 induced by the Wiener structure $\tau$. Then $\left(6, d_{\tau}\right)$ is a metric space. We assume that $\left(\mathbb{Q}, d_{\tau}\right)$ is connected and separable. Therefore there exist a countable number of points $\left\{p_{k} ; k=1,2, \ldots\right\}$ such that $\left\{W\left(p_{k}\right) ; k=1,2, \ldots\right\}$ is a covering of $C Q$ and $W\left(p_{k}\right) \cap\left(\bigcup_{j=1}^{k-1} W\left(p_{j}\right)\right) \neq \emptyset$ for all $k \geq 2$. For the sake of simplicity, let $W_{k} \equiv W\left(p_{k}\right)$ and $\phi_{k} \equiv \phi_{\left(p_{k}\right)}, k \geq 1$.

We will define a path $X$ on $W_{1} \cup W_{2}$ in the following three steps:

Step (1). Suppose $\mathfrak{X}(0)=p_{0} \in W_{1}$. Let $X_{1}$ be the local diffusion in $\phi_{1}\left(W_{1}\right)$ starting at $\phi_{1}\left(p_{0}\right)$ constructed in $(A)$ by using the standard Wiener process $W(t)$ in (15). Let $\rho_{1}$ be the exit time of $X_{1}$ from $\phi_{1}\left(W_{1}\right)$. Define

$$
\mathfrak{X}(t)=\phi_{1}^{-1}\left(X_{1}(t)\right), \quad t \leq \rho_{1} .
$$

Now, if (i) $\rho_{1}=\infty$ or (ii) $\rho_{1}<\infty$ and $\mathfrak{X}\left(\rho_{1}\right) \in \partial\left(W_{1} \cup W_{2}\right)$ then we put $\rho_{2}=$ $\rho_{3}=\cdots=0$.

Step (2). Suppose $\rho_{1}<\infty$ and $X\left(\rho_{1}\right) \in W_{2}^{0}$, the interior of $W_{2}$. Take the Wiener process $W\left(t+\rho_{1}\right)-W\left(\rho_{1}\right)$ in (15) and let $X_{2}$ be the corresponding local diffusion in $\phi_{2}\left(W_{2}\right)$ starting at $\phi_{2}\left(\nVdash\left(\rho_{1}\right)\right)$. Let $\rho_{2}$ be the exit time of $X_{2}$ from $\phi_{2}\left(W_{2}\right)$. Define

$$
\mathfrak{X}(t)=\phi_{2}^{-1}\left(X_{2}\left(t-\rho_{1}\right)\right), \quad \rho_{1} \leq t \leq \rho_{1}+\rho_{2} .
$$

If (i) $\rho_{2}=\infty$ or (ii) $\rho_{2}<\infty$ and $\mathfrak{X}\left(\rho_{1}+\rho_{2}\right) \in \partial\left(W_{1} \cup W_{2}\right)$, then we put $\rho_{3}=\rho_{4}$ $=\cdots=0$.

Step (3). Suppose $\rho_{2}<\infty$ and $X\left(\rho_{1}+\rho_{2}\right) \in W_{1}^{0}$. Take the Wiener process $W\left(t+\rho_{2}\right)-W\left(\rho_{2}\right)$ in (15) and let $X_{3}$ be the corresponding local diffusion in $\phi_{1}\left(W_{1}\right)$ starting at $\phi_{1}\left(X\left(\rho_{1}+\rho_{2}\right)\right)$. Let $\rho_{3}$ be the exit time of $X_{3}$ from $\phi_{1}\left(W_{1}\right)$. Define

$$
\mathfrak{X}(t)=\phi_{1}^{-1}\left(X_{3}\left(t-\rho_{1}-\rho_{2}\right)\right), \quad \rho_{1}+\rho_{2} \leq t \leq \rho_{1}+\rho_{2}+\rho_{3} .
$$

Repeating the previous procedure, we end up with a process $\chi$ in $W_{1} \cup W_{2}$ defined up to the "explosion time" $\rho=\rho_{1}+\rho_{2}+\cdots$. Using Ito's formula of the second type and the transformation rule of $(A, \sigma)$, we can show easily the following

Lemma III.1. Let $(U, \psi)$ be a chart with $U \subset W_{1} \cup W_{2}$. Suppose $\zeta$ is a stopping time such that $\zeta<\rho$ and $\mathfrak{X}(\zeta) \in U$. Let $\rho_{0}$ be the exit time of $X^{\zeta}(t) \equiv$ $\mathfrak{X}(t+\zeta)$ from $U$. Then, for $t<\rho_{0}$, 


$$
X(t) \equiv \psi\left(\chi^{\zeta}(t)\right)=X(0)+\int_{0}^{t} A(X(s)) d W(s)+\int_{0}^{t} \sigma(X(s)) d s,
$$

where $W(s)$ is a Wiener process.

Remark. The only trick in showing this lemma is the following: Let $\xi$ be a nonanticipating transformation (see [9]) such that $\left.\xi\right|_{H}: H \rightarrow H$ is unitary. Then $\int_{0}^{t} \xi(s, \omega) d W(s, \omega)$ is also a Wiener process. We sketch the proof as follows. First observe that a process $X(t)$ in $B$ is a Wiener process if and only if $|A d X(t)|^{2}$ $=\|A\|_{2}^{2} d t$ for all Hilbert-Schmidt operators $A$ of $H$. Let $X(t)=\int_{0}^{t} \xi(s, \omega) d W(s, \omega)$, where $W$ is a Wiener process, then $|A d X(t)|^{2}=|A \xi(t) d W(t)|^{2}=\|A \xi(t)\|_{2}^{2} d t$ by Lemma 3.2 of [9]. But $\|A \xi(t)\|_{2}^{2}=\left\|(A \xi(t))(A \xi(t))^{*}\right\|_{1}=\left\|A \xi(t) \xi(t)^{*} A^{*}\right\|_{1}=\left\|A A^{*}\right\|_{1}$ $=\|A\|_{2}^{2}$, since $\xi(t)$ is unitary. Therefore, $|A d X(t)|^{2}=\|A\|_{2}^{2} d t$ for all HilbertSchmidt operators $A$ of $H$.

We now need an a priori bound. The bound in the following lemma is weaker than that in [10, p. 93]. However, it is easier to prove and is enough for our later discussion. Let $A$ and $\sigma$ be given by Theorem 5.1 of [9] and $\|A(x)-I\|_{2} \leq K$, $|\sigma(x)| \leq K$ for all $x$ in $B$.

Lemma III.2. Suppose $X(t)$ is the solution of the stochastic integral equation

$$
X(t)=x_{0}+\int_{0}^{t} A(X(s)) d W(s)+\int_{0}^{t} \sigma(X(s)) d s .
$$

Let $\rho$ be the exit time of $X(t)$ from $\left\{x \in B ;\left\|x-x_{0}\right\|<r\right\}, r>0$. Then Prob $\{\rho \leq \epsilon\}$ $=o(\epsilon)$ as $\epsilon \rightarrow 0$. In fact Prob $\{\rho \leq \epsilon\} \leq$ constant $\times \epsilon^{2}$ for small $\epsilon>0$, where the constant does not depend on $\epsilon$.

Proof. Obviously

$$
\operatorname{Prob}\{\rho \leq \epsilon\}=\operatorname{Prob}\left\{\sup _{0 \leq t \leq \epsilon}\left\|X(t)-x_{0}\right\| \geq r\right\} .
$$

Thus our assertion is

$$
\operatorname{Prob}\left\{\sup _{0 \leq t \leq \epsilon}\left\|X(t)-x_{0}\right\| \geq r\right\}=o(\epsilon) \quad \text { as } \epsilon \rightarrow 0 .
$$

Without loss of generality, we may assume $x_{0}=0$. By our assumption, the function $\eta(x)=\|x\|^{2}$ is $C^{2}$. It is easy to see that $\left\|\eta^{\prime}(x)\right\|_{B^{*}}=2\|x\|,\left\|\eta^{\prime}(x)-\eta^{\prime}(y)\right\|_{B^{*}} \leq$ $2\|x-y\|$ and $\left\|\eta^{\prime \prime}(x)\right\|_{B, B^{*}} \leq 2$. For the sake of easy reading, we let $X_{s} \equiv X(s)$ and $W_{s} \equiv W(s)$ in the following proof.

Apply Ito's formula of the first type to $\eta$ to obtain

But

$$
\begin{aligned}
\eta\left(X_{t}\right)= & \int_{0}^{t}\left(A^{*}\left(X_{s}\right) \eta^{\prime}\left(X_{s}\right), d W_{s}\right) \\
& +\int_{0}^{t}\left\{\left\langle\eta^{\prime}\left(X_{s}\right), \sigma\left(X_{s}\right)\right\rangle+1 / 2 \text { trace }\left[A^{*}\left(X_{s}\right) \eta^{\prime \prime}\left(X_{s}\right) A\left(X_{s}\right)\right]\right\} d s .
\end{aligned}
$$




$$
\begin{aligned}
\mid \int_{0}^{t}\left\langle\eta^{\prime}\left(X_{s}\right)\right. & \left., \sigma\left(X_{s}\right)\right\rangle d s\left|\leq \int_{0}^{t}\right| \eta^{\prime}\left(X_{s}\right) \| \sigma\left(X_{s}\right) \mid d s \\
& \leq K \beta \int_{0}^{t}\left\|\eta^{\prime}\left(X_{s}\right)\right\|_{B}{ }^{*} d s=2 K \beta \int_{0}^{t}\left\|X_{s}\right\| d s \\
& \leq 2 K \beta \int_{0}^{\epsilon}\left\|X_{s}\right\| d s \quad \text { if } t \leq \epsilon ;
\end{aligned}
$$

and

$$
\begin{aligned}
&\left|\int_{0}^{t} \operatorname{trace} A^{*}\left(X_{s}\right) \eta^{\prime \prime}\left(X_{s}\right) A\left(X_{s}\right) d s\right| \\
& \leq \int_{0}^{t}\left\|A^{*}\left(X_{s}\right)\right\|_{H, H}\left\|\eta^{\prime \prime}\left(X_{s}\right)\right\|_{1}\left\|A\left(X_{s}\right)\right\|_{H, H} d s \\
& \leq \int_{0}^{t} 2(1+K)^{2} \int_{B}\|y\|^{2} p_{1}(d y) d s \\
& \leq 2 \epsilon(1+K)^{2} \int_{B}\|y\|^{2} p_{1}(d y), \quad t \leq \epsilon,
\end{aligned}
$$

since

$$
\begin{aligned}
\left\|A^{*}(x)\right\|_{H, H} & =\|A(x)\|_{H, H} \\
& \leq 1+\|A(x)-I\|_{H, H} \leq 1+\|A(x)-I\|_{2} \leq 1+K,
\end{aligned}
$$

and

$$
\left\|\eta^{\prime \prime}(x)\right\|_{1} \leq \int_{B}\|y\|^{2} p_{1}(d y)\left\|\eta^{\prime \prime}(x)\right\|_{B, B^{*}} \text { for all } x \in B
$$

Let $\epsilon>0$ be so small that $\epsilon(1+K)^{2} \int_{B}\|y\|^{2} p_{1}(d y)<r^{2} / 2$. Then from (16) we have, for $t \leq \epsilon$,

$$
\left\|X_{t}\right\|^{2} \leq\left|\int_{0}^{t}\left(A^{*}\left(X_{s}\right) \eta^{\prime}\left(X_{s}\right), d W_{s}\right)\right|+2 K \beta \int_{0}^{\epsilon}\left\|X_{s}\right\| d s+\frac{r^{2}}{2}
$$

or

$$
\left\|X_{t}\right\|^{2}-\frac{r^{2}}{2} \leq\left|\int_{0}^{t}\left(A^{*}\left(X_{s}\right) \eta^{\prime}\left(X_{s}\right), d W_{s}\right)\right|+2 K \beta \int_{0}^{\epsilon}\left\|X_{s}\right\| d s
$$

Hence

$$
\begin{aligned}
\operatorname{Prob} & \left\{\sup _{0 \leq t \leq \epsilon}\left\|X_{t}\right\| \geq r\right\}=\operatorname{Prob}\left\{\sup _{0 \leq t \leq \epsilon}\left\|X_{t}\right\|^{2} \geq r^{2}\right\} \\
(17) & \leq \operatorname{Prob}\left\{\sup _{0 \leq t \leq \epsilon}\left|J_{0}\left(A^{*}\left(X_{s}\right) \eta^{\prime}\left(X_{s}\right), d W_{s}\right)\right|+2 K \beta \int_{0}^{\epsilon}\left\|X_{s}\right\| d s \geq \frac{r^{2}}{2}\right\} \\
& \left.\leq \operatorname{Prob}\left\{\sup _{0 \leq t \leq \epsilon}\left|\int_{0}^{t}\left(A^{*}\left(X_{s}\right) \eta^{\prime}\left(X_{s}\right), d W_{s}\right)\right| \geq \frac{r^{2}}{4}\right\}+\operatorname{Prob} 2 K \beta \int_{0}^{\epsilon}\left\|X_{s}\right\| d s \geq \frac{r^{2}}{4}\right\}
\end{aligned}
$$

Now, apply (3) and (4) of [9, Theorem 3.2$]$ to get the estimate of the first term in the last inequality: 
1972]

$$
\begin{aligned}
\operatorname{Prob}\left\{\sup _{0 \leq t \leq \epsilon}\left|\int_{0}^{t}\left(A^{*}\left(X_{s}\right) \eta^{\prime}\left(X_{s}\right), d W_{s}\right)\right| \geq \frac{r^{2}}{4}\right\} \\
\leq \frac{16}{r^{4}} \varepsilon\left[\left\{\int_{0}^{\epsilon}\left(A^{*}\left(X_{s}\right) \eta^{\prime}\left(X_{s}\right), d W_{s}\right)\right\}^{2}\right] \\
\quad=\frac{16}{r^{4}} E \int_{0}^{\epsilon}\left|A^{*}\left(X_{s}\right) \eta^{\prime}\left(X_{s}\right)\right|^{2} d s \\
\leq \frac{16}{r^{4}}(1+K)^{2} \beta^{2} \mathcal{E} \int_{0}^{\epsilon}\left\|\eta^{\prime}\left(X_{s}\right)\right\|_{B}^{2} d s \\
=\frac{64}{r^{4}}(1+K)^{2} \beta^{2} \mathcal{E} \int_{0}^{\epsilon}\left\|X_{s}\right\|^{2} d s=c_{1} \mathscr{E} \int_{0}^{\epsilon}\left\|X_{s}\right\|^{2} d s .
\end{aligned}
$$

On the other hand, apply Čebyšev's inequality to the last term of (17) to get

$$
\begin{aligned}
& \operatorname{Prob}\left\{2 K \beta \int_{0}^{\epsilon}\left\|X_{s}\right\| d s \geq \frac{r^{2}}{4}\right\} \leq \frac{64 K^{2} \beta^{2}}{r^{4}} \varepsilon\left[\left\{\int_{0}^{\epsilon}\left\|X_{s}\right\| d s\right\}^{2}\right] \\
& \leq \frac{64 K^{2} \beta^{2} \epsilon}{r^{4}} \mathscr{E} \int_{0}^{\epsilon}\left\|X_{s}\right\|^{2} d s \\
& \leq \frac{64 K^{2} \beta^{2}}{r^{4}} \mathcal{E} \int_{0}^{\epsilon}\left\|X_{s}\right\|^{2} d s, \quad \epsilon<1, \text { say }, \\
& =c_{2} \varepsilon \int_{0}^{\epsilon}\left\|X_{s}\right\|^{2} d s \text {. }
\end{aligned}
$$

Putting (18) and (19) into (17), we get immediately

$$
\operatorname{Prob}\left\{\sup _{0 \leq t \leq \epsilon}\left\|X_{t}\right\| \geq r\right\} \leq c \mathcal{E} \int_{0}^{\epsilon}\left\|X_{s}\right\|^{2} d s
$$

where $c=c_{1}+c_{2}$.

Finally, we consider the given stochastic integral equation

$$
X_{s}=\int_{0}^{s} A\left(X_{u}\right) d W_{u}+\int_{0}^{s} \sigma\left(X_{u}\right) d u=W_{s}+\int_{0}^{s}\left(A\left(X_{u}\right)-I\right) d W_{u}+\int_{0}^{s} \sigma\left(X_{u}\right) d u
$$

It can be checked easily that

$$
\xi\left(\left\|X_{s}\right\|^{2}\right) \leq a s
$$

where $\alpha$ is a constant depending only on $K, \beta$ and the quantity $\int_{B}\|y\|^{2} p_{1}(d y)$.

Evidently we finish the proof by putting (21) into (20). Q.E.D.

Let us return to the process $\mathfrak{X}(t)$ in $W_{1} \cup W_{2}$ defined up to the "explosion time" $\rho$.

Lemma III.3. If $\rho<\infty$ then $\mathfrak{X}(\rho-)$ exists and belongs to $\partial\left(W_{1} \cup W_{2}\right)$.

Proof. Let $D_{1}=\partial W_{1}-\partial\left(W_{1} \cup W_{2}\right)$ and $D_{2}=\partial W_{2}-\partial\left(W_{1} \cup W_{2}\right)$. 
Let $\zeta_{1}<\zeta_{2}<\zeta_{3}<\cdots<\rho$ be the successive hitting time of $D_{1}, D_{2}, D_{1}, D_{2}, \cdots$. Let $D_{1}^{(n)}$ and $D_{2}^{(n)}$ be two increasing sequences of Borel sets converging to $D_{1}$ and $D_{2}$ respectively such that $d_{\tau}\left(D_{1}^{(n)}, D_{2}^{(n)}\right)=\epsilon^{(n)} \rightarrow 0$ as $n \rightarrow \infty$. Let $E_{n}$ be the event $\left\{\zeta_{2 j-1} \in D_{1}^{(n)}\right.$ and $\zeta_{2 j} \in D_{2}^{(n)}$ for all $\left.j=1,2, \ldots\right\}$. To finish the proof it is sufficient to show that $\operatorname{Prob}\left(E_{n}\right)=0$ for all $n \geq 1$.

Now by Lemma III.2, Prob $\left\{\zeta_{j}-\zeta_{j-1} \leq 1 / j \mid \zeta_{j-1}<\infty\right\} \leq$ constant $\times 1 / j^{2}$. If $\operatorname{Prob}\left(E_{n}\right)>0$ for some $n$ then on the even $E_{n}$ we have $\rho>$ tail of $\sum_{j=1}^{\infty} 1 / j=\infty$ by an application of the first Borel-Cantelli lemma. But this contradicts the assumption $\rho<\infty$. Therefore $\operatorname{Prob}\left(E_{n}\right)=0$ for all $n \geq 1$. Q.E.D.

Let $X_{2}(t), t<\rho_{2}$, be the process in $W_{1} \cup W_{2}$ constructed before. Let $X_{3}(t)$ be the local diffusion in $\phi_{3}\left(W_{3}\right)$. Using $X_{2}$ and $X_{3}$ in place of $X_{1}=\phi_{1}^{-1}\left(X_{1}\right)$ and $X_{2}$, we can construct a process $X_{3}(t), t<\rho_{3}$, in $W_{1} \cup W_{2} \cup W_{3}$ in the same manner. The process $\mathfrak{X}_{3}$ has the same properties as those in Lemma III.1 and Lemma III.3 for $\mathfrak{X}_{2}$ : namely, $\mathfrak{X}_{3}$ is defined up to $\rho_{3}$, it is compatible with local diffusions on charts of $W_{1} \cup W_{2} \cup W_{3}$, and $X_{3}\left(\rho_{3}-\right) \in \partial\left(W_{1} \cup W_{2} \cup W_{3}\right)$ if $\rho_{3}<\infty$. Inductively, for each $n$ we can construct a process $\mathfrak{X}_{n}(t)$, defined up to time $\rho_{n}$, in $\bigcup_{j=1}^{n} W_{j}$ with the same properties in Lemma III.1 and Lemma III.3.

Finally we define a process $X(t)$ in $W 0$ up to explosion time $\rho=\lim _{k \rightarrow \infty} \rho_{k}$ by $\mathfrak{X}(t)=\mathfrak{X}_{n}(t), t<\rho_{n}$. Note that $\rho_{1}<\rho_{2}<\cdots<\rho$. $X(t)$ is unambiguously defined since, for each $k \geq 1, \mathfrak{X}_{k-1}(t)=\mathfrak{X}_{k}(t)$ up to $t<\rho_{k-1}$. It can be checked easily that $\mathfrak{X}(t)$ begins afresh at its stopping times. Moreover, $X(t)$ solves the stochastic differential equation $d \mathfrak{X}(t, \omega)=A(\mathfrak{X}(t, \omega)) d W(t, \omega)+\sigma(\mathfrak{X}(t, \omega)) d t$ in the sense of the following

Theorem III.1. Let $(U, \phi)$ be a chart in 60 . Suppose $\zeta$ is a stopping time of $\mathfrak{X}$ such that $\zeta<\rho$ and $\mathfrak{X}(\zeta) \in U$. Let $\rho_{0}$ be the exit time of $\mathfrak{X}^{\zeta}(t) \equiv \mathfrak{X}(t+\zeta)$ from $U$. Then, for $t<\rho_{0}$,

$$
X(t) \equiv \phi\left(X^{\zeta}(t)\right)=X(0)+\int_{0}^{t} A(X(s)) d W(s)+\int_{0}^{t} \sigma(X(s)) d s,
$$

where $W(s)$ is a Wiener process.

(C) The infinitesimal generator.

Theorem III.2. Let $f$ be a bounded function of class $C^{2}$ on 6 . Let $\mathfrak{X}_{p}(t)$ denote the process constructed above starting at $p$. Then

$$
\lim _{t \downarrow 0} \frac{E\left[f\left(X_{p}(t)\right)\right]-f(p)}{t}=1 / 2 \operatorname{trace} A^{*}(x) f_{\phi}^{\prime \prime}(x) A(x)+\left\langle\sigma(x), f_{\phi}^{\prime}(x)\right\rangle,
$$

where $\mathcal{E}$ is the expectation with respect to the standard Wiener process (cf. [9]), $x=\phi(p), f_{\phi}=f \circ \phi^{-1}$ and $\phi$ is a chart at $p$.

Remark. The right-hand side of (22) is independent of the chart $\phi$. This can 
be seen easily by using the transformation rule of $(A, \sigma)$. This differential operator with $(A, \sigma)$ given by Proposition III.1 will be called the Beltrami-Laplace operator of 70 . It coincides with the usual one if 60 is a finite dimensional Riemannian manifold and with the Laplacian introduced by Gross [5] if 28 is $B$.

Proof. Let $(U, \phi)$ be a chart at $p$ such that $\phi(U)$ is an open ball around $\phi(p)$. Let $\rho_{0}$ be the exit time of $\mathfrak{X}_{p}(t)$ from $U$. Then

$$
\mathcal{E}\left[f\left(\mathfrak{X}_{p}(t)\right)\right]=E\left[f\left(\mathfrak{X}_{p}(t)\right) \cdot 1_{t<\rho_{0}}\right]+\mathcal{E}\left[f\left(\mathfrak{X}_{p}(t)\right) \cdot 1_{\rho_{0} \leq t}\right]
$$

where $1_{E}$ indicates the characteristic function of the event $E$.

But $E\left[f\left(X_{p}(t)\right) \cdot 1_{\rho_{0} \leq t}\right] \leq\|f\|_{\infty} \operatorname{Prob}\left\{\rho_{0} \leq t\right\}=o(t)$ by Lemma III.2. Therefore

$$
\lim _{t 10} \frac{\mathcal{E}\left[f\left(\mathfrak{X}_{p}(t)\right)\right]-f(p)}{t}=\lim _{t 10} \frac{\mathcal{E}\left[f\left(\mathfrak{X}_{p}(t)\right) \cdot 1_{t<p_{0}}\right]-f(p)}{t} .
$$

Now by Theorem III. 1 on the event $t<\rho_{0}$ the process $X(t) \equiv \phi\left(\mathfrak{X}_{p}(t)\right)$ satisfies the equation

$$
X(t)=\phi(p)+\int_{0}^{t} A(X(s)) d W(s)+\int_{0}^{t} \sigma(X(s)) d s .
$$

Apply Ito's formula of the first kind to $f_{\phi}$ :

$$
\begin{aligned}
f_{\phi}(X(t))-f_{\phi}(\phi(p))= & \int_{0}^{t}\left(A^{*}(X(s)) f_{\phi}^{\prime}(X(s)), d W(s)\right) \\
& +\int_{0}^{t}\left\{\left\langle f_{\phi}^{\prime}(X(s)), \sigma(X(s))\right\rangle\right. \\
& \left.\quad+1 / 2 \operatorname{trace} A^{*}(X(s)) f_{\phi}^{\prime \prime}(X(s)) A(X(s))\right\} d s .
\end{aligned}
$$

Taking expectation on both sides and using (4) of [Q, Theorem 3.2], we obtain immediately that

$$
\begin{aligned}
& \frac{E\left[f_{\phi}(X(t))\right]-f(p)}{t}=\frac{1}{t} \xi \int_{0}^{t}\left\{\left\langle f_{\phi}^{\prime}(X(s)), \sigma(X(s))\right\rangle\right. \\
&\left.+1 / 2 \text { race } A^{*}(X(s)) f_{\phi}^{\prime \prime}(X(s)) A(X(s))\right\} d s \\
& \rightarrow 1 / 2 \operatorname{trace} A^{*}(x) f_{\phi}^{\prime \prime}(x) A(x)+\left\langle\sigma(x), f_{\phi}^{\prime}(x)\right\rangle \text { as } t \rightarrow 0,
\end{aligned}
$$

where $x=\phi(p)$. The theorem follows by combining (23) and (24). Q.E.D.

IV. Brownian motion on a Riemann-Wiener manifold. From now on 60 will denote a connected and separable $C^{k}$-Riemann-Wiener manifold $(k \geq 3)$. Let $\left(A_{g}, \sigma_{g}\right)$ be defined in 20 by $A_{g}(x)=\bar{g}(x)^{-1 / 2}, \sigma_{g}(x)=-1 / 2$ sp $\widetilde{\Gamma}(x) \circ\left[\bar{g}(x)^{-1 / 2} \times \bar{g}(x)^{-1 / 2}\right]$. By Proposition III.1, $\left(A_{g}, \sigma_{g}\right)$ are diffusion coefficients. Moreover, $\left(A_{g}, \sigma_{g}\right)$ are locally Lipschitzian because they are Fréchet differentiable. To see this simply recall that $\left[8\right.$, Definition II.4] and the definition of $\widetilde{\Gamma}$ imply that $A_{g}(\cdot)-I$ and $\widetilde{\Gamma}(\cdot) \circ$ $\left[\bar{g}(\cdot)^{-1 / 2} \times \bar{g}^{\left.(\cdot)^{-1 / 2}\right]}\right.$ are Fréchet differentiable maps into $\mathcal{B}\left(B, B^{*}\right)$ and $\mathcal{B}\left(B, B ; B^{*}\right)$, respec- 
tively. The following lemma concludes that $\sigma_{g}$ is differentiable.

Lemma IV.1. If $\Phi$ is a Fréchet differentiable transformation from an open subset $U$ of $B$ into the Banach space $\mathcal{B}\left(B, B ; B^{*}\right)$, then $\mathrm{sp} \Phi$ is also Fréchet differentiable from $U$ into $H$. Moreover, $(\operatorname{sp} \Phi)^{\prime}(x) u=\operatorname{sp}\left[\Phi^{\prime}(x) u\right]$ for $x \in U$ and $u \in B$.

Proof. Simply note that $\Phi^{\prime}(x) u \in \mathfrak{B}\left(B, B ; B^{*}\right)$ for all $x \in U$ and $u \in B$. Q.E.D.

Let $B(t)$ denote the process constructed in the previous section corresponding to the locally Lipschitzian diffusion coefficients $\left(A_{g}, \sigma_{g}\right)$. We will call $\mathscr{B}(t)$ a Brownian motion in 6 . Note that $B(t)$ is completely determined by the Riemannian structure $g$. For each point $p \in \mathbb{W}, B_{p}(t)$ denotes the motion $B(t)$ starting at $p$. Let $\beta_{t}(p, \cdot)$ denote the transition probabilities of $\mathbb{B}(t)$, i.e. $\beta_{t}(p, E)=\operatorname{Prob}\left\{\mathbb{B}_{p}(t) \in E\right\}$. We will study the spatial homogeneity of $\mathbb{B}(t)$ and the relation between $\beta_{t}(p, \cdot)$ and the local measures $q_{t}(p, \cdot)$ defined in [8].

6 has a metric $d_{\tau}$ induced by its Wiener structure $\tau$. Thus we can define isometries with respect to $d_{\tau}$ in the usual way. However, the group of $d_{\tau}$-isometries is not the one with respect to which $B(t)$ is spatially homogeneous. On the other hand, 6 has an almost-metric $d_{g}$ (in the sense that two points in 70 may have infinite distance) induced by its Riemannian structure $g$. For a more detailed discussion of $d_{g}$ we refer the reader to [8]. We will define $d_{g}$-isometries and show that $B(t)$ is spatially homogeneous with respect to the group of $d_{g}$-isometries.

Definition IV.1. A surjective map $J$ from $W$ into itself is said to be $d_{g}$-isometric if it is at least $C^{2}$-diffeomorphic with respect to $\tau$ and $d_{g}(J x, J y)=d_{g}(x, y)$ for all $x$ and $y$ in 60 .

Remark. We review briefly some material from [8]. For each point $x$ in 6 , $\left(R_{x}, T_{x}((9))\right.$ is an abstract Wiener space with inner product $g(x)$ for $R_{x}$ and norm $\tau(x)$ for $T_{x}((9))$. Let $|\cdot|_{x}$ denote the norm of $R_{x}$ corresponding to $g(x)$. Moreover, for each point $x$ in $W$ there exists a $C^{\infty}$-Riemannian manifold $(R(x), g)$ containing $x$ such that $T_{y}(R(x))=R_{y}$ for all $y \in R(x)$. If $J$ is a $d_{g}$-isometry, then $y \in R(x)$ if and only if $J(y) \in R(J(x))$ because $d_{g}(J x, J y)<\infty$ if and only if $d_{g}(x, y)<\infty$.

Proposition IV.1. Suppose $J$ is a $C^{j}$-diffeomorphism $(j \geq 2)$ from 6 onto itself with respect to $\tau$. Then $J$ is a $d_{g}$-isometry if and only if, for each $x \in \mathbb{W}, J^{\prime}(x)\left(R_{x}\right)$ $C R_{J(x)}$ and $J^{\prime}(x)$ is a unitary operator from $R_{x}$ into $R_{J(x)}$.

Proof of sufficiency. Note first that $J^{\prime}(x)\left(R_{x}\right)=R_{J(x)}$ because $J^{\prime}(x)$ is a uni-


is nonsingular, where $c$ denotes complement. It follows easily that

$$
\left|J^{\prime}(x) u\right|_{J(x)}=|u|_{x} \text { for all } u \in T_{x}\left(i^{9}\right) \text {. }
$$

Recall that we used the convention $|u|_{x}=\infty$ if $\left.u \in T_{x}(6)\right) \cap R_{x}^{c}$ in [8] in defining the almost-metric $d_{g}$.

Let $x$ and $y$ be any two points in 60 . Let $r$ be a piecewise differentiable curve 
connecting $x$ and $y$, i.e. $r(0)=x, r(1)=y$. The $L_{g}$-length of $r$ is defined in [8] by $L_{g}(r)=\int_{0}^{1}\left|r^{\prime}(t)\right|_{r(t)} d t$. It follows from (25) that $L_{g}(J \circ r)=L_{g}(r)$. Thus $d_{g}(x, y)=$ inf $L_{g}(r)=\inf _{r} L_{g}(J \circ r) \geq d_{g}(J x, J y)$. Conversely, $d_{g}(J x, J y) \geq d_{g}(x, y)$ because $J$ is onto. Hence, $d_{g}(J x, J y)=d_{g}(x, y)$ for all $x$ and $y$ in 6.

Proof of necessity. Let $u \in T_{\boldsymbol{x}}(\mathbb{C})$ and let $r$ be any piecewise differentiable curve in 68 such that $r(0)=x$ and $r^{\prime}(0)=u$. Then $|u|_{x}=\lim _{t 10}(1 / t) d_{g}(x, r(t))$. On the other hand, the curve $J \circ r$ is such that $J \circ r(0)=J(x),(J \circ r)^{\prime}(0)=J^{\prime}(x) u$. Thus $\left|J^{\prime}(x) u\right|_{J(x)}=\lim _{t 10}(1 / t) d_{g}(J(x), J \circ r(t))$. But by assumption $d_{g}(J(x), J \circ r(t))$ $=d_{g}(x, r(t))$. Therefore, $\left|J^{\prime}(x) u\right|_{J(x)}=\lim _{t \downarrow 0}(1 / t) d_{g}(x, r(t))=|u|_{x}$. Hence if $u \in R_{x}$ then $|u|_{x}<\infty$ and $\left|J^{\prime}(x) u\right|_{J(x)}<\infty$, hence $J^{\prime}(x) u \in R_{J(x)^{\circ}}$. Thus $J^{\prime}(x)\left(R_{x}\right) \subset R_{J(x)}$ and $J^{\prime}(x)$ is an isometry. It can be checked easily that $J^{\prime}(x)\left(R_{x}\right)=R_{J(x)}$ by the assumption that $J^{\prime}(x)$ is nonsingular from $T_{x}(79)$ into $T_{J(x)}((9))$. Therefore, $J^{\prime}(x)$ is a unitary operator from $R_{x}$ into $R_{J(x)}$ Q.E.D.

Remark. In $[8, \oint$ II.e] we show that if $r$ is a curve in 6 such that $r(0)=x$ and $r^{\prime}(0)=u \in R_{x}$ then $r(t) \in R(x)$ for small $t$. Thus $|u|_{x}<\infty$ if and only if $d_{g}(x, r(t))<\infty$ for small $t$. On the contrary, $u \in R_{x}^{c} \cap T_{x}\left(t^{8}\right)$ if and only if $r(t) \epsilon$ $R(x)^{c}$. Thus $|u|_{x}=\infty$ iff $d_{g}(x, r(t))=\infty$ for small $t$.

Proposition IV.2. Suppose $J$ is a $d_{g}$-isometry of 6 . Let $(U, \phi)$ and $(V, \psi)$ be two charts of 10 sucb that $J$ is $C^{2}$-diffeomorphic from $U$ onto $V$. Let $J_{\phi, \psi} \equiv$ $\psi \mathrm{J} \phi^{-1}$. Then for all $x \in \phi(U) \subset B$ we bave

$$
\begin{aligned}
\bar{g}\left(J_{\phi, \psi} x\right)^{-1} & =J_{\phi, \psi}^{\prime}(x) \bar{g}(x)^{-1} J_{\phi, \psi}^{\prime}(x)^{*}, \\
\sigma_{g}\left(J_{\phi, \psi} x\right) & =J_{\phi, \psi}^{\prime}(x)\left(\sigma_{g}(x)\right)+1 / 2 \operatorname{sp} J_{\phi, \psi}^{\prime \prime}(x) \circ\left[A_{g}(x) \times A_{g}(x)\right] .
\end{aligned}
$$

Remark. For every $x$ in $\phi(U), J_{\phi, \psi}^{\prime \prime}(x) \circ\left[A_{g}(x) \times A_{g}(x)\right]$ is a spur operator of $H$. This will be shown in the proof.

Proof. It follows from Proposition IV.1 that, for each $y \in U,\langle u, v\rangle_{y}=$ $\left\langle J^{\prime}(y) u, J^{\prime}(y) v\right\rangle_{J(y)}$ holds for all $u, v \in R_{y^{*}}$. This is equivalent to saying that, for each $x$ $\epsilon \phi(U),\langle\bar{g}(x) b, k\rangle=\left\langle\bar{g}\left(J_{\phi, \psi} x\right) J_{\phi, \psi}^{\prime}(x) b, J_{\phi, \psi}^{\prime}(x) k\right\rangle$ holds for all $b, k \in H$. Therefore we have

$$
\bar{g}(x)=J_{\phi, \psi}^{\prime}(x)^{*} \circ \bar{g}\left(J_{\phi, \psi} x\right) \circ J_{\phi, \psi}^{\prime}(x) .
$$

This implies that $\bar{g}\left(J_{\phi, \psi} x\right)^{-1}=J_{\phi, \psi}^{\prime}(x) \bar{g}(x)^{-1} J_{\phi, \psi}^{\prime}(x)^{*}$ for all $x$ in $\phi(U)$. Note that (26) is similar to (12) in Proposition III.1. Thus by the same computation and argument we can obtain easily that

$$
\begin{aligned}
\tilde{\Gamma}\left(J_{\phi, \psi} x\right) \circ\left[\bar{g}\left(J_{\phi, \psi} x\right)^{-1 / 2}\right. & \left.\times \bar{g}\left(J_{\phi, \psi} x\right)^{-1 / 2}\right] \\
& \quad-J_{\phi, \psi}^{\prime}(x) \circ \tilde{\Gamma}(x) \circ\left[\bar{g}(x)^{-1 / 2} \times \bar{g}(x)^{-1 / 2}\right] \circ[S(x) \times S(x)] \\
& =-J_{\phi, \psi}^{\prime \prime}(x) \circ\left[\bar{g}(x)^{-1 / 2} \times \bar{g}(x)^{-1 / 2}\right] \circ[S(x) \times S(x)],
\end{aligned}
$$


where $S(x)$ is given by $A_{g}\left(J_{\phi, \psi} x\right)=J_{\phi, \psi}^{\prime}(x) A_{g}(x) S(x)$ as in Remark 2 following Definition III.1. But we know that the left-hand side of the above identity is a spur operator. Therefore, $J_{\phi, \psi}^{\prime \prime}(x) \circ\left[\bar{g}(x)^{-1 / 2} \times \bar{g}(x)^{-1 / 2}\right]$ is also a spur operator since $S(x)$ is a unitary operator of $H$ and $\sigma_{g}\left(J_{\phi, \psi} x\right)-J_{\phi, \psi}^{\prime}(x)\left(\sigma_{g}(x)\right)=1 / 2$ sp $J_{\phi, \psi}^{\prime \prime}(x) \circ$ $\left[A_{g}(x) \times A_{g}(x)\right]$ by taking - $1 / 2$ sp on both sides. Hence

$$
\sigma_{\boldsymbol{g}}\left(J_{\phi, \psi} x\right)=J_{\phi, \psi}^{\prime}(x)\left(\sigma_{\boldsymbol{g}}(x)\right)+1 / 2 \operatorname{sp} J_{\phi, \psi}^{\prime \prime}(x) \circ\left[A_{\boldsymbol{g}}(x) \times A_{\boldsymbol{g}}(x)\right] \text { Q.E.D. }
$$

Theorem IV.1 (Spatial homogeneity). Let $\beta_{t}(p, \cdot)$ be the transition probabilities of $B(t)$. If $J$ is a $d_{g}$-isometry of 6 then $\beta_{t}(J p, J E)=\beta_{t}(p, E)$ for all $t>0$, $p \in \mathbb{C}$ and all Borel subsets $E$ of 70 .

Proof. Let $B^{J}(t) \equiv J \mathfrak{B}(t)$. Let $\mathfrak{B}_{p}(t)$ be the process $\mathbb{B}(t)$ starting at $p \in \mathbb{W}$. Then $\mathfrak{B}_{p}^{J}(t) \equiv J \mathscr{B}_{p}(t)$ is a process starting at $J(p)$. Let $(U, \phi)$ and $(V, \psi)$ be charts at $p$ and $J(p)$ respectively such that $J$ is $C^{2}$-diffeomorphic from $U$ onto $V$. Let $J_{\phi, \psi} \equiv \psi J \phi^{-1}$ as in Proposition IV.2. Let $\rho_{0}$ be the exit time of $B_{p}(t)$ from $U$. Then $\rho_{0}$ is also the exit time of $\mathbb{B}_{p}^{J}(t)$ from $V$. Let $X(t) \equiv \phi\left(\mathscr{B}_{p}(t)\right)$ and $Y(t) \equiv \psi\left(\mathbb{B}_{p}^{J}(t)\right)$. Then $Y(t)=\psi J \phi^{-1}\left(\phi \mathbb{B}_{p}(t)\right)=J_{\phi, \psi}(X(t))$. It follows from Theorem III. 1 that

$$
X(t)=X(0)+\int_{0}^{t} A_{g}(X(s)) d W(s)+\int_{0}^{t} \sigma_{g}(X(s)) d s, \quad t<\rho_{0} .
$$

Apply Ito's formula of the second type to get

$$
\begin{aligned}
J_{\phi, \psi}(X(t))= & J_{\phi, \psi}(X(0))+\int_{0}^{t} J_{\phi, \psi}^{\prime}(X(s)) \circ A_{g}(X(s)) d W(s) \\
& +\int_{0}^{t}\left\{J_{\phi, \psi}^{\prime}(X(s))\left(\sigma_{g}(X(s))\right)\right. \\
& \left.\quad+1 / 2 \operatorname{sp} J_{\phi, \psi}^{\prime \prime}(X(s)) \circ\left[A_{g}(X(s)) \times A_{g}(X(s))\right]\right\} d s .
\end{aligned}
$$

Now, we use Proposition IV.2 and obtain immediately that

$$
\begin{aligned}
Y(t)= & \psi(J(p))+\int_{0}^{t} A_{g}\left(J_{\phi, \psi} X(s)\right) S^{-1}(X(s)) d W(s) \\
& +\int_{0}^{t} \sigma_{g}\left(J_{\phi, \psi} X(s)\right) d s,
\end{aligned}
$$

where $S(x)$ is given in the proof of Proposition IV.2, i.e.

$$
Y(t)=\psi(J(p))+\int_{0}^{t} A_{\boldsymbol{g}}(Y(s)) d W^{\prime}(s)+\int_{0}^{t} \sigma_{\boldsymbol{g}}(Y(s)) d s,
$$

where $W^{\prime}(s)=\int_{0}^{s} s^{-1}(X(u)) d W(u)$ is a Wiener process (cf. the remark below Lemma III.1). On the other hand, $\psi B_{J(p)}(t)$ also satisfies the above stochastic integral equation. Thus $Y(t)=\psi^{\Re} J(p)(t)$ by the uniqueness of solution. Therefore, $\mathfrak{B}_{p}^{J}(t)=\mathfrak{B}_{J(p)}(t)$. It follows that for any Borel subset $E$ of $W$ we have 


$$
\begin{aligned}
B_{t}(J p, J E) & =\operatorname{Prob}\left\{B_{J(p)}(t) \in J E\right\}=\operatorname{Prob}\left\{\mathscr{B}_{p}^{J}(t) \in J E\right\} \\
& =\operatorname{Prob}\left\{J \mathbb{B}_{p}(t) \in J E\right\}=\operatorname{Prob}\left\{\mathbb{B}_{p}(t) \in E\right\}=\beta_{t}(p, E) . \quad \text { Q.E.D. }
\end{aligned}
$$

In the rest of this paper we will compare results in this paper with those in [8]. Recall that for each $x$ in $(d)$ there exists an open neighborhood $U(x)$ of $x$ such that $\exp _{x}: \exp _{x}^{-1}(U(x)) \subset T_{x}\left(\mathbb{B}^{8}\right) \rightarrow U(x)$ is $C^{1}$-diffeomorphic. A local measure with parameter $t>0$ at $x$ is defined by $q_{t}(x, E)=p_{t}^{(x)}\left(0, \exp _{x}^{-1}(E)\right)$, in which $E$ is a Borel subset of $U(x)$ and $p_{t}^{(x)}$ is Wiener measure in $T_{x}(W)$. In $\$ 0$ of the introduction to [8] we remarked that the local measures $\left\{q_{t}(x,).\right\}$ are local first order approximations to the transition probabilities of a Brownian motion. This will be shown in the following theorem. We will also study the equivalence-perpendicularity relation between them.

Theorem IV.2. (i) Let $x$ be any fixed point in 6 . Then for any Borel subset $E$ of $U(x)$,

$$
\lim _{t ! 0} \frac{1}{t^{\alpha}}\left|\beta_{t}(x, E)-q_{t}(x, E)\right|=0, \text { where } 0 \leq \alpha<1 / 2
$$

(ii) Let $x_{0} \in 20$ and let $U$ be a subdomain of a chart such that $U \supset U\left(x_{0}\right)$. If $x$ is in $U$ then $\beta_{t}(x, \cdot)$ and $q_{s}\left(x_{0}, \cdot\right)$, as measures in $U\left(x_{0}\right)$, are equivalent if and only if $t=s$ and $d_{g}\left(x, x_{0}\right)<\infty$. Otherwise they are mutually singular.

Remark. We conjecture that $\beta_{t}(x, \cdot)$ and $\beta_{s}(y, \cdot)$ are equivalent if and only if $t=s$ and $d_{g}(x, y)<\infty$ and that they are mutually singular otherwise.

Proof of (i). Let $\rho$ be the exit time of $B_{x}(t)$ from $U(x)$ and let $\theta_{t}(x, E)=$ $\left.\operatorname{Prob}_{\{}(t) \in E, t<\rho\right\}$. Let $E$ be any Borel subset of $U(x)$; then $0 \leq \beta_{t}(x, E)-$ $\theta_{t}(x, E) \leq \operatorname{Prob}\{\rho \leq t\}=o(t)$ by Lemma III.2. Thus to show (i) it is sufficient to prove that, for $0 \leq \alpha<1 / 2$,

$$
\lim _{t \prod_{1}} \frac{1}{t^{a}}\left|\theta_{t}(x, E)-q_{t}(x, E)\right|=0 .
$$

On the other hand, if $a>0$ then $p_{t}^{(x)}\left(0,\left\{u \in T_{x}\left(\hat{(A)}^{\mathrm{n}}\right) ; \tau(x)(u)>a\right\}\right)=o\left(t^{n}\right)$ for any integer $n \geq 1$ by Fernique's theorem [3]. This remark and Lemma III. 2 show that we need only prove (27) for $E$ of the form $E=\left\{y \in \mathbb{l} ; d_{\tau}(x, y)<a\right\} \subset U(x)$, where $a>0$ is small.

Recall that $U(x)$ is contained in a chart $(U, \phi)$ at $x$. Let $X(t) \equiv \phi\left(\mathbb{B}_{x}(t)\right)$, $t<\rho$. Then

$$
X(t)=\phi(x)+\int_{0}^{t} A_{g}(X(s)) d W(s)+\int_{0}^{t} \sigma_{g}(X(s)) d s .
$$

Piech [11] constructs a fundamental solution $\left\{r_{t}(y, \cdot)\right\}$ of the parabolic equation 


$$
\partial u(t, y) / \partial t=1 / 2 \text { trace } A_{\boldsymbol{g}}^{*}(y) u_{y y}(t, y) A_{\boldsymbol{g}}(y)+\left\langle\sigma_{\boldsymbol{g}}(y), u_{y}(t, y)\right\rangle .
$$

It can be checked that

$$
\lim _{t\rceil 0} \frac{1}{t^{\alpha}}\left|r_{t}(\phi(x), \phi(E))-p_{t}(\phi(x), \phi(E))\right|=0,
$$

where $0 \leq \alpha<1 / 2$ and $p_{t}$ is Wiener measure in $B$ with parameter $t>0$. Moreover, by using the same idea as in [13, Theorem 3] we can show that $r_{t}(\phi(x), \phi(E))=$ $\theta_{t}(x, E)$. On the other hand, let $r_{t}^{(\phi)}(x, \cdot)$ be defined, as in [8], by

$$
r_{t}^{(\phi)}(x, E)=p_{t}\left(0, \phi_{*, x} \circ \exp _{x}^{-1}(E)\right)
$$

Then it can be checked easily that

$$
\lim _{t \downarrow 0} \frac{1}{t^{\alpha}}\left|q_{t}(x, E)-r_{t}^{(\phi)}(x, E)\right|=0 .
$$

Therefore we end up having to show that

$$
\lim _{t 10} \frac{1}{\alpha}\left|p_{t}(\phi(x), \phi(E))-r_{t}^{(\phi)}(x, E)\right|=0
$$

in order to finish the proof of the assertion (i). Let $T=\phi_{*, x}{ }^{\circ} \exp _{x}^{-1} \circ \phi^{-1} \circ$ $l_{\phi(x)}$, where $l_{\phi(x)}$ is the translation by $\phi(x)$, i.e. $l_{\phi(x)}(y)=y+\phi(x), y \in B$. Then it is easy to check that $T$ satisfies the assumption of Proposition II. 1 and the conclusion there implies (29).

Proof of (ii). Let $(U, \phi)$ be a chart with $U$ given in (ii). Define $r_{s}^{(\phi)}\left(x_{0}, \cdot\right)$ by (28). Then we show in the proof of [8, Lemma III.3] that $q_{s}\left(x_{0}, \cdot\right)$ and $r_{s}^{(\phi)}\left(x_{0}, \cdot\right)$, as Borel measures in $U\left(x_{0}\right)$, are equivalent, i.e.

$$
q_{s}\left(x_{0}, \cdot\right) \approx r_{s}^{(\phi)}\left(x_{0}, \cdot\right) \text { in } U\left(x_{0}\right)
$$

Let $\rho$ be the exit time of $B_{x}(t)$ from $U$ and let $\theta_{t}(x, \cdot)$ be defined by $\theta_{t}(x, D)$ $=\operatorname{Prob}\left\{B_{x}(t) \in D, t<\rho\right\}$ in which $D \in$ Borel field of $U\left(x_{0}\right)$. Then

$$
\beta_{t}(x, \cdot) \approx \theta_{t}(x, \cdot) \text { in } U\left(x_{0}\right)
$$

as in the finite dimensional case. tion

Let $X(t) \equiv \phi\left(B_{x}(t)\right), t<\rho$. Then $X(t)$ satisfies the stochastic integral equa-

$$
X(t)=\phi(x)+\int_{0}^{t} A_{g}(X(s)) d W(s)+\int_{0}^{t} \sigma_{\mathcal{g}}(X(s)) d s .
$$

The corresponding parabolic equation is

$$
\partial u(t, y) \prime^{\prime} \partial t-1 / 2 \operatorname{trace} A_{g}^{*}(y) u_{y y}(t, y) A_{g}(y)+\left\langle\sigma_{g}(y), u_{y}(t, y)\right\rangle .
$$


Let $\left\{r_{t}(y, \cdot)\right\}$ be the fundamental solution of the above equation constructed by Piech [11] and note that $A_{g}$ is nonsingular. It can be checked that "absolutely continuous" in Theorem 1 of [12] can be replaced by "equivalent". Therefore, $r_{t}(y, \cdot) \approx p_{t}(y, \cdot)$ in $B$. On the other hand, $\theta_{t}(x, D)=r_{t}(\phi(x), \phi(D))$ by the remark in the proof of (i). Hence we have

$$
\theta_{t}(x, \cdot) \approx p_{t}(\phi(x), \phi(.)) \text { in } U\left(x_{0}\right) .
$$

Finally, let $T=\phi_{*, x_{0}} \circ \exp _{x_{0}}^{-1} \circ \phi^{-1} \circ l_{\phi(x)}$, where $l_{\phi(x)}$ is the translation by $\phi(x)$. If $d_{g}\left(x, x_{0}\right)<\infty$ then $\phi(x)-\phi\left(x_{0}\right) \in H$ and it can be checked easily that $T$ is admissible. Therefore, if $d_{s}\left(x, x_{0}\right)<\infty$, then $p_{t}(\phi(x), \phi(\cdot)) \approx$ $r_{s}^{(\phi)}\left(x_{0}, \cdot\right)$ if and only if $t=s$. Otherwise, they are mutually singular. If $d_{g}\left(x, x_{0}\right)$ $=\infty$ then obviously $p_{t}(\phi(x), \phi(\cdot))$ and $r_{s}^{(\phi)}\left(x_{0}, \cdot\right)$ are mutually singular for any $t_{3} s>0$. The results in this paragraph and the relations (30)-(32) give our assertion (ii). Q.E.D.

\section{BIBLIOGRAPHY}

1. Ju. L. Dalec'kiǐ and Ja. I. Šnằderman, Diffusion and quasiinvariant measures on infinite-dimensional Lie groups, Funkciónal. Anal. i Priložen. 3 (1969), no. 2, 88-90. (Russian) MR 40 \#2138.

2. J. Eells and K. D. Elworthy, Wiener integration on certain Banach manifolds, Some Problems in Non-Linear Analysis, Centro Internazionale Matematico Estivo, 1970, pp. 6794.

3. X. Fernique, Intégrabilité des vecteurs Gaussiens, C. R. Acad. Sci. Paris Sér. A-B 270 (1970), A1698-A1699.

4. L. Gross, Abstract Wiener spaces, Proc. Fifth Berkeley Sympos. Math. Statist. and Probability, vol. II: Contributions to Probability Theory, part 1, Univ. of California Press, Berkeley, Calif., 1967, pp. 31-42. MR 35 \#3027.

5. - Potential theory on Hilbert space, J. Functional Analysis 1 (1967), 123181. MR $37 \# 3331$.

6. K. Ito, Stochastic differential equations in a differentiable manifold, Nagoya Math. J. 1 (1950), 35-47. MR 12, 425.

7. —, Brownian motions in a Lie group, Proc. Japan Acad. 26 (1950), 4-10. MR 13, 760 .

8. H.-H. Kuo, Integration theory on infinite dimensional manifolds, Trans. Amer. Math. Soc. 159 (1971), 57-78.

9. - Stochastic integrals in abstract Wiener space, Pacific J. Math. 40 (1972).

10. H. P. McKean, Jr., Stochastic integrals, Probability and Math. Statist., no. 5, Academic Press, New York and London, 1969. MR 40 \#947.

11. M. Ann Piech, A fundamental solution of the parabolic equation on Hilbert space, J. Functional Analysis 3 (1969), 85-114. MR 40 \#4815.

12. - Some regularity properties of diffusion processes on abstract Wiener space, J. Functional Analysis 8 (1971), 153-172.

13. H.-H. Kuo and M. Ann Piech, Stochastic integrals and parabolic equations in abstract Wiener space (to appear).

COURANT INSTITUTE OF MATHEMATICAL SCIENCES, NEW YORK UNIVERSITY, NEW YORK, NEW YORK 10012

Current address: Department of Mathematics, University of Virginia, Charlottesville, Virginia 22903 